\title{
EDITORIAL
}

\section{SNPs and prognosis of GvHD before HCT: any progress?}

Bone Marrow Transplantation (2016) 51, 161-162; doi:10.1038/ bmt.2015.240; published online 12 October 2015

Antigen disparity at the level of $\mathrm{MHC}$ is a well-recognized risk factor for acute GvHD, ${ }^{1}$ but this does not explain the entire story. In transplantations that are MHC matched but minor histocompatibility antigen ( $\mathrm{mHA}$ ) mismatch disparate, donor $\mathrm{T}$ cells recognize MHC peptides derived from the products of recipient polymorphic genes, the mHAs. ${ }^{2,3}$ The expression of $\mathrm{mHAs}$ is wide and variable. Thus, different mHAs might dictate variable phenotype, target organ involvement, development kinetics of GvHD or antitumor responses after allogeneic hematopoietic cell transplantation $(\mathrm{HCT}){ }^{4}$ Some $\mathrm{mHAs}$, such as HA-1, HA-2, HB-1 and $B C L 2 A 1$, are primarily found on hematopoietic cells, whereas others such as the $\mathrm{H}-\mathrm{Y}$ antigens, HA-3, HA-8 and UGT2B17 are ubiquitous.

Single-nucleotide polymorphisms (SNPs) are single base-pair changes and are ubiquitous across the genome in coding, noncoding and untranslated regions. Such variants are strong candidates for disease susceptibility mutations, and gene localization studies screen large numbers of SNPs to test the cooccurrence of SNP alleles and disease: an individual carrying one or two copies of a high-risk variant is at increased risk of developing a disease. ${ }^{5}$

In 2003, Murata et al. ${ }^{3}$ used cDNA expression cloning to identify a novel $\mathrm{mHA}$ encoded by UGT2B17, an autosomal gene in the multigene UDP-glycosyltransferase 2 family that is selectively expressed in liver, intestine and APCs. UGT2B17 is immunogenic because of differential expression of the protein in donor and recipient cells as a consequence of a homozygous gene deletion in the donor. The Japanese group then looked at the association between UGT2B17 deletion and the incidence of acute GvHD in their cohort of 435 patients. Contrary to their expectations, they did not find an association with acute GvHD but they suggested the use of a UGT2B17-positive donor was an independent risk factor for higher transplant-related mortality and lower survival after transplantation. ${ }^{6}$

In contrast, McCarroll et al. ${ }^{7}$ analyzed three HCT cohorts with a total of $1345 \mathrm{HLA}$-identical sibling donor-recipient pairs, and they found the risk of acute GvHD was greater (odds ratio $=2.5 ; 95 \%$ confidence interval 1.4-4.6) when donor and recipient were mismatched for the homozygous deletion of UGT2B17 (donor (-), recipient (+)). However, they discuss that this is comparable to the established effect of sex mismatch (female donor, male recipient), and hence UGT2B17 mismatches cannot explain a comparable fraction of GvHD incidence due to the lower frequency at which UGT2B17 mismatches arise between siblings. Therefore, there was a need to confirm this effect by independent, multicenter investigations, and that it may not extend to transplants involving unrelated donors.

In this issue of Bone Marrow Transplantation, Santos et al. ${ }^{8}$ look at UGT2B17 mismatch in their cohort of 1127 recipients receiving $\mathrm{HCT}$ from an HLA-identical sibling donor. They performed UGT2B17 genotyping by sequence-specific primed PCR as previously described. ${ }^{9}$ They found a UGT2B17 mismatch was present in $6.1 \%$ of cases. Incidence of severe acute GvHD was higher in the UGT2B17 mismatched pairs (22.7\% vs $14.6 \%)$, but was not statistically significant in multivariate analysis. Similarly, they could not detect differences in chronic GvHD, overall survival, relapse-free survival, transplant-related mortality or relapse. Nor did donor genotype alone predict outcome as Terakura et al. ${ }^{6}$ previously reported.

However, the investigators extended their study with unplanned analyses and found an interesting and provocative result: the impact of UGT2B17 disparity on grade III-IV acute GvHD was revealed when the immune dominant $\mathrm{H}-\mathrm{Y}$ antigen effect was removed by studying male donors alone $(25.1 \%$ vs $12.8 \%$; $P=0.005)$. This association was confirmed by the multivariate analysis ( $P=0.024$; hazard ratio $2.25,95 \%$ confidence interval $1.11-$ 4.57). Overall survival was also worse for this group $(P=0.005)$.

These findings suggest the clinical impact disparity at different mHAs may have hierarchical relationships. The dependence for $\mathrm{mHA}$ display and TCR recognition by MHC provides a structurefunction explanation for the severe GvHD consequences of HLA mismatch relative to individual $\mathrm{mHA}$. It has been long recognized that sex-mismatch transplantation has strong predictable clinical consequences resulting from alloimmune T- and B-lymphocyte responses against a family of $Y$-chromosome-encoded $\mathrm{mHA}$ called $\mathrm{H}-\mathrm{Y}$ antigens. ${ }^{10}$ The strong clinical impact of sex-mismatched transplantation likely results from $\mathrm{Y}$-chromosome transmission providing disparity across nine or more $\mathrm{H}-\mathrm{Y}$ antigens. In fact, detection of allogeneic antibodies against multiple $\mathrm{H}-\mathrm{Y}$ antigens 3 months following sex-mismatched transplantation $(F \rightarrow M$ HCT) seems to have additive clinical consequences. The detection of antibodies against four or more $\mathrm{H}-\mathrm{Y}$ antigens predicted chronic GvHD and nonrelapse mortality with hazard ratios 5-fold and 20fold, respectively, greater than $\mathrm{H}-\mathrm{Y}$ seronegative recipients. ${ }^{11}$ In the current study, Santos et al. show the clinical impact of UGT2B17 deletion is significant when considering only male donors who presumably have no $\mathrm{H}-\mathrm{Y}$ immunity. Disparity for other individual autosomal $\mathrm{mHA}$ loci such as $\mathrm{HA}-1$ have shown weaker clinical effects relative to $\mathrm{H}-\mathrm{Y}$ antigens. ${ }^{12}$ Other explanations for UGT2B17 disparity being more potent in male recipients will need to be considered. In their discussion, the authors also entertain the idea that UGT2B17-positive recipients may metabolize cyclosporine A (CSA) faster than patients who lack UGT2B17, and this may explain the higher incidence of GvHD. Almost $90 \%$ of their entire cohort received CSA. It would be interesting to look at UGT2B17 mismatch in other cohorts not treated with CSA.

Our understanding of the immunogenicity of $\mathrm{mHA}$ and tumorassociated antigens and their role in preventing disease relapse and development of GvHD is evolving rapidly. Thus far, mHAs have been predominately coding SNP single amino acid changes, but UGT2B17 presents a null allele mutation (Table 1).

Some of the issues with defining genetic risk for GvHD in HCT include the low replication rate of positive findings in other cohorts that may be related to various study designs or analytic approaches, or differences in genetic variants frequencies in different populations. In the case of UGT2B17, the estimate of homozygous deletion can vary from $4 \%$ (African ancestry) to $12 \%$ (Europeans ancestry) to $72 \%$ (Japanese/Chinese ancestry). ${ }^{13-15}$ In a recent validation study evaluating published SNPs associated with acute GvHD, the Fred Hutchinson group achieved a replication rate of only $7 \% .{ }^{16}$ Definitive utilization of SNPs studies and more adequately powered studies will require extensive multicenter participation, resources to prospectively collect and analyze pretransplant DNA, standardized definition of clinical phenotypes and informed consent appropriate for performing genome-wide genetic studies. Ideally, these studies should also include 
Table 1. Currently identified minor histocompatibility antigens and their immunogenic mechanisms

\begin{tabular}{llll}
\hline Antigen & Peptide sequence & MHC restriction & Immunogenic mechanism \\
\hline HA1 & VLR/HDDLLEA & $\left(A^{*} 0201\right)$ & HLA binding difference \\
HA2 & YIGEVLVSV/M & $\left(A^{*} 0201\right)$ & TCR binding difference \\
HA3 & VT/MEPGTAQY & $\left(A^{*} 0101\right)$ & Proteasome destruction \\
HA8 & R/PTLKVLEV & $\left(A^{*} 0201\right)$ & Allele restricted proteolysis \\
HB1 & EEKRGSLH/YVW & $(B 44)$ & Biallelic CTL recognition \\
BCL2A1 & DYLQY/CVLQI & $\left(A^{*} 2402\right)$ & One autosome gene with two T-cell epitopes \\
UGT2B17 & KEFEDD/GIINW & $\left(B^{*} 4403\right)$ & Homozygous gene deletion \\
\hline Abbreviations: $C T L=$ cytotoxic & T lymphocyte; TCR =T-cell receptor. & $\left(A^{*} 2902\right)$ & \\
\hline
\end{tabular}

proteomic studies to link the genotype and phenotype that may open the door to a better understanding of the mechanism and development of therapeutics as it has done for cardiovascular disease risk. ${ }^{17}$

\section{CONFLICT OF INTEREST}

Dr Paczesny is an inventor on a patent on 'Methods of detection of graft-versus-host disease' (US-13/573,766). The authors declare no conflict of interest.

MI Abu Zaid ${ }^{1}$, S Paczesny ${ }^{1,2}$ and DB Miklos ${ }^{3}$ ${ }^{1}$ Indiana University School of Medicine, Indiana University Melvin and Bren Simon Cancer Center, Indianapolis, IN, USA;

${ }^{2}$ Department of Pediatrics, Microbiology and Immunology, Indiana University School of Medicine, Melvin and Bren Simon Cancer Center, Indianapolis, IN, USA and ${ }^{3}$ Division of Blood and Marrow Transplantation, Department of Medicine, Stanford University Medical Center, Stanford, CA, USA E-mail: sophpacz@iu.edu ordmiklos@stanford.edu

\section{REFERENCES}

1 Flomenberg N, Baxter-Lowe LA, Confer D, Fernandez-Vina M, Filipovich A, Horowitz $\mathrm{M}$ et al. Impact of HLA class I and class II high-resolution matching on outcomes of unrelated donor bone marrow transplantation: HLA-C mismatching is associated with a strong adverse effect on transplantation outcome. Blood 2004; 104: 1923-1930.

2 Goulmy E, Schipper R, Pool J, Blokland E, Falkenburg JH, Vossen J et al. Mismatches of minor histocompatibility antigens between HLA-identical donors and recipients and the development of graft-versus-host disease after bone marrow transplantation. N Engl J Med 1996; 334: 281-285.

3 Murata M, Warren EH, Riddell SR. A human minor histocompatibility antigen resulting from differential expression due to a gene deletion. $J$ Exp Med 2003; 197: 1279-1289.

4 Kaplan DH, Anderson BE, McNiff JM, Jain D, Shlomchik MJ, Shlomchik WD. Target antigens determine graft-versus-host disease phenotype. J Immunol 2004; 173: 5467-5475.
5 Lewis CM. Genetic association studies: design, analysis and interpretation. Brief Bioinform 2002; 3: 146-153.

6 Terakura S, Murata M, Nishida T, Emi N, Akatsuka Y, Riddell SR et al. A UGT2B17positive donor is a risk factor for higher transplant-related mortality and lower survival after bone marrow transplantation. Br J Haematol 2005; 129: 221-228.

7 McCarroll SA, Bradner JE, Turpeinen H, Volin L, Martin PJ, Chilewski SD et al. Donor-recipient mismatch for common gene deletion polymorphisms in graftversus-host disease. Nat Genet 2009; 41: 1341-1344.

8 Santos N, Rodríguez-Romanos R, Nieto JB, Buño I, Vallejo C, Jiménez-Velasco A et al. UGT2B17 minor histocompatibility mismatch and clinical outcome after HLA-identical sibling donor stem cell transplantation. Bone Marrow Transplant 2016; 51: 79-82.

9 Spierings $\mathrm{E}$, Kim $\mathrm{YH}$, Hendriks M, Borst E, Sergeant R, Canossi A et al. Multicenter analyses demonstrate significant clinical effects of minor histocompatibility antigens on GvHD and GvL after HLA-matched related and unrelated hematopoietic stem cell transplantation. Biol Blood Marrow Transplant 2013; 19: 1244-1253.

10 Popli R, Sahaf B, Nakasone H, Lee JY, Miklos DB. Clinical impact of H-Y alloimmunity. Immunol Res 2014; 58: 249-258.

11 Nakasone H, Tian L, Sahaf B, Kawase T, Schoenrock K, Perloff S et al. Allogeneic HY antibodies detected 3 months after female-to-male HCT predict chronic GVHD and nonrelapse mortality in humans. Blood 2015; 125: 3193-3201.

12 Hansen JA, Chien JW, Warren EH, Zhao LP, Martin PJ. Defining genetic risk for graft-versus-host disease and mortality following allogeneic hematopoietic stem cell transplantation. Curr Opin Hematol 2010; 17: 483-492.

13 Xue $Y$, Sun D, Daly A, Yang F, Zhou X, Zhao M et al. Adaptive evolution of UGT2B17 copy-number variation. Am J Hum Genet 2008; 83: 337-346.

14 Redon R, Ishikawa S, Fitch KR, Feuk L, Perry GH, Andrews TD et al. Global variation in copy number in the human genome. Nature 2006; 444: 444-454.

15 Spierings E, Hendriks M, Absi L, Canossi A, Chhaya S, Crowley J et al. Phenotype frequencies of autosomal minor histocompatibility antigens display significant differences among populations. PLoS Genet 2007; 3: e103.

16 Chien JW, Zhang XC, Fan W, Wang H, Zhao LP, Martin PJ et al. Evaluation of published single nucleotide polymorphisms associated with acute GVHD. Blood 2012; 119: 5311-5319.

17 Ho JE, Chen WY, Chen MH, Larson MG, McCabe EL, Cheng S et al. Common genetic variation at the IL1RL1 locus regulates IL-33/ST2 signaling. J Clin Invest 2013; 123: 4208-4218. 